\title{
Immune Function in Mania
}

\author{
Ziad Kronfol and J. Daniel House
}

\section{Introduction}

There is now a large body of evidence suggesting a close interaction between the central nervous system and the immune system. More specifically, immunological abnormalities have been described in relation to psychological stress (Jemmott and Locke 1984) and in patients with psychiatric illness (DeLisi and Crow 1986). We have earlier reported a decrease in mitogen-induced lymphocyte proliferation in hospitalized depressed patients compared to normal controls (Kronfol et al. 1983). These findings have been replicated by independent investigators (Schleifer et al. 1984). Negative life events and depression have been associated with immunological impairment, as evidenced by decreased responsivity to mitogen stimulation and reduced natural killer cell activity (Bartrop et al. 1977; Locke et al. 1984). On the other hand, to the best of our knowledge, the immune status of patients with manic illness has never been investigated. We now report an impairment in immune regulation in manic patients similar to what has been described in patients with major depression.

From the Department of Psychiatry, University of Michigan Medical Center, Ann Arbor, MI (Z.K.), and the Department of Family Practice, University of Iowa College of Medicine, Iowa City, IA (J.D.H.).

Supported in part by the Nellie Ball Trust Fund and NIMH Grant MH-42988.

Address reprint requests to Dr. Z. Kronfol, Department of Psychiatry, University of Michigan Medical Center, UH-9C, 1500 E. Medical Center Drive, Box 0120, Ann Arbor, MI 481090120.

Received August 22, 1987; revised November 13, 1987.

\section{Methods}

\section{Subjects}

Three groups of subjects were identified: acutely manic patients $(n=11)$, deteriorated schizophrenic controls ( $n=22$ ), and normal controls $(n=37)$. Both the manic patients and the schizophrenic patients were hospitalized at the University of Iowa Psychiatric Hospital. Psychiatric diagnoses were made in accordance with DSM-III criteria. Mean ages (+ SD) for the three groups were as follows: manic patients $36.5 \pm 13.9$, schizophrenic controls $30.0 \pm$ 8.8, and normal controls $31.3 \pm 9.0$. These differences were not statistically significant ( $F$ $=1.68, p \mathrm{NS}$ ) The sex distribution of the three groups was as follows: manic patients 6 men, 5 women, schizophrenic controls 18 men, 4 women, normal controls 19 men, 18 women. These differences in sex distribution were not statistically significant either $\left(x^{2}=5.66, p\right.$ NS). Patients and controls were free of medical illness or drugs known to interfere with immune function. Psychotropic medications, however, were not considered as exclusion criteria. The immunological assays on both the manic patients and the schizophrenic controls were done while these patients were acutely ill, usually within 10 days of admission to the hospital. Normal controls were healthy volunteers who were free of medical and psychiatric illness and were not taking medications at the time of the study.

\section{Procedures}

Twenty milliliters of blood was drawn from each subject between 8:00 and 9:00 AM. Whenever 
possible, blood samples from subjects in all three groups were assayed together to control for dayto-day variation in the assay. As this was not always possible, a blood sample from a normal control was therefore included in every assay. A total white blood cell (WBC) count and a differential were done using standard procedures. Blood was then centrifuged over FicollHypaque and the lymphocytes were separated for culture or surface marker analysis. Lymphocyte cultures were then established in triplicate, as previously described (Kronfol et al. 1983). To each well, one of the following mitogens was added: no mitogen for background transformations (blank); phytohemagglutin-P (PHA), $2.5 \mu \mathrm{g} / \mathrm{ml}$; concanavalin-A (Con-A), 10 $\mu \mathrm{g} / \mathrm{ml}$; and pokeweed mitogen (PWM), 2.5 $\mu \mathrm{g} / \mathrm{ml}$. The cultures were kept for 4 days prior to addition of tritiated thymidine and harvesting. Tritiated thymidine uptake was then measured with a scintillation counter. Mean scintillation counts per minute $(\mathrm{cpm})$ were calculated for each mitogen, and the three groups were compared. For the surface marker analyses, lymphocytes from each subject were washed several times and incubated separately with one of the following commercially available monoclonal antibodies (Coulter Immunology, Hialeah, FL): $B_{1}$ for total B lymphocytes, $T_{11}$ for total $T$ lymphocytes, $T_{4}$ for helper $T$ lymphocytes, and $T_{8}$ for suppressor $\mathrm{T}$ lymphocytes. The cells were then washed again and incubated with a fluorescein-conjugated second antibody (GAM-FITC; Coulter Immunology). The frequency (percentage) of each cell type was then determined indirectly with fluorescent microscopy. Differences between the three groups were analyzed with analysis of variance (ANOVA). This procedure is relatively powerful with respect to unequal sample sizes. Whenever an overall significance was found (i.e., $p<0.05$ ), pairwise comparison follow-up tests were done using the Duncan multiple range test.

\section{Results}

A comparison of the different immune variables in manic patients, schizophrenic controls, and normal controls is presented in Table 1 . There were no significant differences in either total WBC counts, total lymphocyte counts, or any of the lymphocyte subsets among the three groups. Results of the mitogen stimulation assays, on the other hand, revealed significant reductions in lymphocyte responsivity to the mitogens PHA and Con-A in manic patients compared to both schizophrenic controls and normal controls. However, the latter two groups did not significantly differ from each other. Responses to PWM were lowest in the manic group, but overall significance was not obtained, partly because of the large standard deviation observed in the schizophrenic group.

\section{Discussion}

Our results indicate a decrease in mitogen-induced lymphocyte proliferation in manic patients in comparison to schizophrenic controls and normal controls. Lymphocyte numbers and lymphocyte subsets, on the other hand, did not significantly differ among the groups. The reduction in lymphocyte responsivity to the mitogens PHA and Con-A suggests an impairment in cell-mediated immune response, as these mitogens are known to stimulate mostly $T$ cells. The response to PWM, a mostly B-cell mitogen, although lower in manic patients, was not significantly different from normal controls.

Several factors, such as age, sex, diurnal variation, medical illness, and drugs, should be considered in interpreting our results. There were no significant differences in age or sex distribution among the groups. All blood samples were drawn at the same time of the day to control for possible diurnal variation. Patients with medical illness or drugs known to interfere with immune function were excluded. All manic and schizophrenic patients were hospitalized on the same unit, so the effects of hospitalization and the milieu were the same for these two groups. All but two of the manic patients were on medications: three were on lithium, three were on neuroleptics, and three were on both lithium and neuroleptics. All but six of the schizophrenic controls were also on medications: 14 were on neuroleptics and 2 were on other drugs. Results of the unmedicated manic patients were similar 
Table 1. Immunological Variables in Manic Patients, Schizophrenic Controls, and Normal Controls ${ }^{a}$

\begin{tabular}{lcccc}
\hline & Mania $(\mathrm{n}=11)$ & $\begin{array}{c}\text { Schizophrenic } \\
\text { controls } \\
(\mathbf{n}=22)\end{array}$ & $\begin{array}{c}\text { Normal } \\
\text { controls } \\
(\mathbf{n}=37)\end{array}$ & $p^{b}$ \\
\hline WBC & $7,867 \pm 2,598$ & $7,797 \pm 2.405$ & $6,787 \pm 1,779$ & NS \\
Total lymphocytes & $2,303 \pm 872$ & $2,414 \pm 1,136$ & $2,540 \pm 629$ & NS \\
$B_{1}(\%)$ & $11.1 \pm 5.0$ & $10.3 \pm 6.2$ & $7.6 \pm 5.7$ & NS \\
$\mathrm{T}_{11}(\%)$ & $59.4 \pm 9.7$ & $67.0 \pm 10.7$ & $66.6 \pm 9.5$ & NS \\
$\mathrm{T}_{4}(\%)$ & $41.0 \pm 8.1$ & $41.2 \pm 8.4$ & $39.1 \pm 6.4$ & NS \\
$\mathrm{T}_{8}(\%)$ & $23,2 \pm 4.0$ & $22.2 \pm 8.6$ & $25.3 \pm 6.0$ & NS \\
$\mathrm{T}_{4} \mathrm{~T}_{8}$ & $1.81 \pm 0.48$ & $2.04 \pm 0.71$ & $1.63 \pm 0.48$ & NS \\
Blank & $719 \pm 409$ & $1,032 \pm 406$ & $940 \pm 702$ & NS \\
PHA $(2.5 \mu \mathrm{g} / \mathrm{ml})$ & $121,976 \pm 36,055$ & $164,764 \pm 64,023$ & $177,773 \pm 66,867$ & 0.0416 \\
Con-A $(10 \mu \mathrm{g} / \mathrm{ml})$ & $76,501 \pm 24,534$ & $137,844 \pm 65,032$ & $151,067 \pm 84,373$ & 0.0154 \\
PWM $(2.5 \mu \mathrm{g} / \mathrm{ml})$ & $70,660 \pm 24,399$ & $133,771 \pm 130,061$ & $109,275 \pm 62,559$ & 0.1566 \\
\hline
\end{tabular}

${ }^{\circ}$ Results are expressed as means \pm SD.

bStatistical analyses are done with ANOVA.

to those of the medicated manic patients in that they showed much lower lymphocytic responses than either the unmedicated schizophrenic controls or the normal controls. Mean mitogenic responses to PHA, Con-A, and PWM in the unmedicated manic patients were 106,773, 73,534 , and 84,144 , respectively. The corresponding values in the unmedicated schizophrenic controls were $203,452,156,607$, and 116,008 , respectively. It is therefore unlikely that the differences in mitogenic responses between the groups is the mere result of medications. Rather, we propose that mania is associated with an impairment in cell-mediated immunity as reflected by a reduced in vitro lymphocyte response to mitogen stimulation. The effects of psychotropic medications on the immune system, however, need further investigation.

In summary, we compared the frequency of different lymphocyte subsets and lymphocyte mitogenic activity in manic patients, schizophrenic controls, and normal controls. We found a significant reduction in mitogenic activity in the manic group, which is suggestive of an impairment of cellular immunity in mania. Our sample size, however, is relatively small, and the potential effects of psychotropic medications need further elucidation. These preliminary re- sults should therefore be confirmed in a larger sample with more drug-free manic patients. The mechanism(s) of immunosuppression should also be investigated.

\section{References}

American Psychiatric Association, Committee on Nomenclature and Statistics (1980): Diagnostic and Statistical Manual of Mental Disorders. ed 3. Washington, DC: American Psychiatric Association.

Bartrop RW, Lazarus L, Luckhurst, et al (1977): Depression and lymphncyte function after bereavement. Lancet I:834-836.

DeLisi LE, Crow TJ (1986): Is schizophrenia a viral or immunological disorder? Psychiatr Clin North Am 9:115-132.

Jemmott JB, Locke SE (1984): Psychosocial factors, immunologic mediation, and human susceptibility to infectious illnesses: How much do we know? Psychol Bull 95:78-108.

Kronfol Z, Silva J, Greden J, et al (1983): Impaired lymphocyte function in depressive illness. Life Sci 33:241-247.

Locke SE, Krause L, Leserman J, et al (1984): Life change stress, psychiatric symptoms, and natural killer cell activity. Psychosom Med 46:441-453.

Schleifer SJ, Keller SE, Meyerson AT, et al (1984): Lymphocyte function in major depressive disorder. Arch Gen Psychiatry 41:484-486. 